\title{
La hormonoterapia de reemplazo no fue beneficiosa en mujeres con enfermedad coronaria
}

Heart and Estrogen/progestin Replacement Study (Estudio HERS). Hulley S, Grady D, Bush T et al. JAMA 1998;280:605-613

Objetivo
Determinar si la TRH continua altera el riesgo de eventos vasculares corona-
rios en mujeres postmenopáusicas con enfermedad coronaria establecida.

Diseño

Ensayo clínico randomizado controlado doble ciego.

\section{Lugar}

Estudio multicéntrico, realizado en 20 centros de atención ambulatoria en los EEUU.

\section{Pacientes}

Se incluyeron 2763 mujeres postmenopáusicas con EC establecida, menores de 80 años y no histerectomizadas. La edad promedio fué de 66.7 años. Se consideró como postmenopáusicas a aquellas mujeres de al menos 55 años con 5 años de amenorrea o con un año de amenorrea y FSH mayor a $40 \mathrm{UI} / \mathrm{l}$ o estradiol menor a $25 \mathrm{pg} / \mathrm{ml}$. Cualesquiera de cuatro eventos definió a las mujeres con enfermedad coronaria establecida: infarto agudo de miocardio (IAM), cirugía de revascularización miocárdi$\mathrm{ca}$, angioplastía o evidencia angiográfica de estenosis (50\% o más de la luz) de al menos una arteria coronaria.

\section{Intervención}

Las mujeres fueron randomizadas a recibir diariamente y en forma ciega comprimidos idénticos conteniendo placebo $(n=1383)$ o terapia hormonal combinada ( $n=1380$ ): $0.625 \mathrm{mg}$ de estrógenos equinos conjugados y $2.5 \mathrm{mg}$ de medroxiprogesterona. El tiempo promedio de seguimiento fue de 4.1 años. Luego del 1er año, el $82 \%$ de las mujeres que recibían terapia de reemplazo continuaban bajo tratamiento, al final del 3er año la adherencia se redujo al $75 \%$.

\section{Medición de Resultados Principales}

Resultados Primarios: IAM no fatal y muerte debida a enfermedad coronaria (IAM fatal, muerte súbita [dentro de la hora de iniciados los síntomas], muerte por insuficiencia cardíaca o durante procedimientos de revascula- rización miocárdica, muerte inexplicada no presenciada por terceros)

Resultados Secundarios:

- Cardiovasculares: procedimientos de revascularización miocárdica, angina inestable que requirió internación, insuficiencia cardíaca, TIA o ACV y arteriopatía periférica.

- No cardiovasculares: eventos tromboembólicos venosos, mortalidad global, mortalidad por cáncer, incidencia de cáncer, fracturas y enfermedad vesicular sintomática.

\section{Resultados Principales}

El análisis de los datos se hizo por intención de tratar* . No se hallaron diferencias significativas en los resultados primarios ni en los secundarios cardiavasculares. El Riesgo Relativo para los resultados primarios fue de 0.99 (IC95\%, 0.80-1.22). Tampoco hubo diferencias en otros resultados secundarios (para los cuales el estudio tiene limitado poder): fracturas, cáncer y mortalidad global. Se encontró una tendencia temporal estadísticamente significativa, por la cuál en el grupo TRH, el riesgo de eventos primarios coronarios disminuyó a lo largo del tiempo. De esta forma, al final del primer año se registró un número mayor de eventos CV en el grupo con TRH respecto al grupo placebo (RR 1.52, IC95\% 1.01-2.29), pero al final de los años 4to y 5 to esta situación se invirtió (RR 0.67, IC95\% 0.46-0.98, p 0.05). En el grupo intervención se detectó un aumento significativo de eventos troboembólicos venosos (34 vs. 12; RR, 2.89; IC95\%, 1.50-5.58). El número necesario para dañar (NND)* fue de $250 @$. En el grupo TRH se observó un descenso de la LDL de un 11\% y un $10 \%$ de aumento del $10 \%$ la HDL.

\section{Conclusiones}

El tratamiento con TRH durante un promedio 4.1 años en mujeres posmenopáusicas con enfermedad coronaria establecida no redujo el riesgo de IAM ni de muerte por causas coronarias ni de ningún otro evento CV. La TRH produjo un aumento del riesgo de eventos tromboembólicos venosos y de enfermedad vesicular. Se observó una tendencia favorable en la incidencia de eventos CV en el grupo TRH recién luego de varios años de terapia hormonal.

\section{COMENTARIO}

Numerosos estudios de cohortes y caso - control han reportado una reducción significativa y consistente del riesgo de eventos cardiovasculares (CV) en mujeres postmenopáusicas que utilizaban terapia de reemplazo hormonal (TRH). La reducción relativa, de alrededor de 30 al 40\%, parecía ser estabie tanto en prevención primaria como en prevención secundaria, beneficiando más a las mujeres con un perfil de riesgo CV más desfavorable ${ }^{1-2}$. Las bases fisiopatológicas que sustentaban este efecto describían 2 posibles mecanismos: la acción sobre las lipoproteínas (HDL $\uparrow$ y $L D L \downarrow$ ) y sobre el endotelio, facilitando la vasodilatación y disminuyendo el proceso de ateroesclerosis. El peso de esta evidencia jugó un papel tan importante en los últimos años, que logró prácticamente imponer su recomendación en mujeres postmenopáusicas sin contraindicaciones formales para la TRH.

El presente estudio, el primer ensayo clínico randomizado y controlado (ECRC) sobre TRH, no encuentra reducción de IAM o muerte CV en mujeres con enfermedad coronaria que recibieron TRH comparadas con el grupo placebo y reporta un aumento significativo de los fenómenos tromboembólicos en el grupo tratado. Los autores desarrollan una excelente discusión sobre las posibles explicaciones de esta aparente contradicción. Siempre se habló de que el beneficio de la TRH podía deberse al sesgo de selección *, ya que las mujeres que eligen TRH son más sanas, tienen mejores condiciones de vida y más controles médicos en general, además de mostrar una adherencia poco común en un tratamiento crónico. Aún cuando estos estudios observacionales lograran un detallado análisis estadístico que controlara posibles confundidores, estos sesgos no desaparecían y siempre quedaba espacio para la duda. Los resultados de este excelente ECRC, que ha cuidado todos los detalles metodológicos, son sorprendentes e inesperados ya que la evidencia epidemiológica, hasta ahora, había anticipado un 'indiscutible' beneficio de La TRH. ¿Deberíamos entonces desechar la idea de La TRH como terapia preventiva?

Alqunos detalles del estudio merecen ser comentados: 1) la edad de las participantes fue, en promedio, 10 años mayor a la de la mayoría de los estudios observacionales, 2) El exceso de eventos primarios del grupo TRH ocurrió durante el primer año, a partir del cual la tendencia se revirtió, 3) si bien el grupo TRH sufrió casi 3 veces más eventos tromboembólicos, la diferencia absoluta fue de 4,1/1000 mujeres/año. Las participantes del estudio, por su edad y por el hecho de tener enfermedad vascular establecida, pueden haber tenido más riesgo de trombosis venosa que una población más sana. Dada la reversión de la tendencia desfavorable inicial de los eventos primarios, que fue también acompañada por otros eventos CV secundarios como angina inestable, revascularización e IAM no fatales, se podría especular con el hecho de que el beneficio inducido por la TRH puede tomar más tiempo en manifestarse y que los eventos ocurridos durante el primer año puedan no estar relacionados directamente a la TRH. No sería este el caso de los fenómenos tromboembóLicos ya que su incidencia fue mayor en el grupo TRH a lo largo de los 4 años. Como conclusión, acuerdo con los autores que recomiendan no iniciar TRH como prevención secundaria de enfermedad coronaria. Asimismo, si bien estos resultados abren una preocupante incógnita con respecto a la prevención primaria de eventos $C V$, no hay que olvidar que es una población claramente diferente y que, por ahora, la mejor evidencia disponible muestra beneficios. Espero que los resultados del Women 's Health Initiative, el ECRC sobre prevención primaria y TRH, puedan definir esta controversia.

\section{Dr. Fernando Rubinstein}

Unidad de Medicina Familiar y Preventiva. Hospital Italiano de Buenos Aires. 